\title{
Introducing Ionic-Current Detection for X-Ray Absorption Spectroscopy in Liquid Cells
}

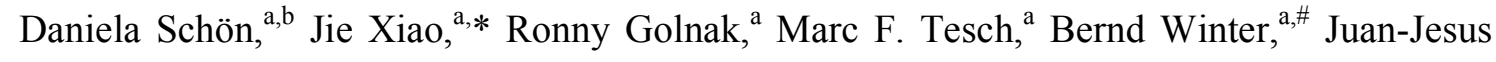
Velasco-Velez, ${ }^{\mathrm{c}}$ Emad F. Aziz ${ }^{\mathrm{a}, \mathrm{b}, \mathrm{d}, *}$

${ }^{\mathrm{a}}$ Institute of Methods for Material Development, Helmholtz-Zentrum Berlin für Materialien und Energie, Albert-Einstein-Strasse 15, 12489 Berlin, Germany

${ }^{b}$ Department of Physics, Freie Universität Berlin, Arnimallee 14, 14195 Berlin, Germany

${ }^{\mathrm{c}}$ Fritz-Haber-Institut der Max-Planck-Gesellschaft, Faradayweg 4-6, 14195 Berlin, Germany

${ }^{\mathrm{d}}$ School of Chemistry, Monash University, Victoria 3800, Australia

*Corresponding authors: jie.xiao@helmholtz-berlin.de; emad.aziz@helmholtz-berlin.de

${ }^{\#}$ Current address: Fritz-Haber-Institut der Max-Planck-Gesellschaft, Faradayweg 4-6, 14195

Berlin, Germany 


\begin{abstract}
Photons and electrons are two common relaxation products upon X-ray absorption, enabling fluorescence yield and electron yield detections for X-ray absorption spectroscopy (XAS). The ions that are created during the electron yield process are relaxation products too, which are exploited in this study to produce ion yield for XA detection. The ionic currents measured in a liquid cell filled with water or iron(III) nitrate aqueous solutions exhibit characteristic $\mathrm{O}$ $\mathrm{K}$-edge and $\mathrm{Fe}$ L-edge absorption profiles as a function of excitation energy. Application of two electrodes installed in the cell is crucial for obtaining the XA spectra of the liquids behind membranes. Using a single electrode can only probe the species adsorbed on the membrane surface. The ionic-current detection, termed as total ion yield (TIY) in this study, also produces an undistorted Fe L-edge XA spectrum, indicating its promising role as a novel detection method for XAS studies in liquid cells.
\end{abstract}

Key words: total ion yield (TIY), ionic current, liquid flow-cell, total electron yield (TEY), $\mathrm{X}$-ray absorption spectroscopy (XAS)

\title{
TOC Graphic
}

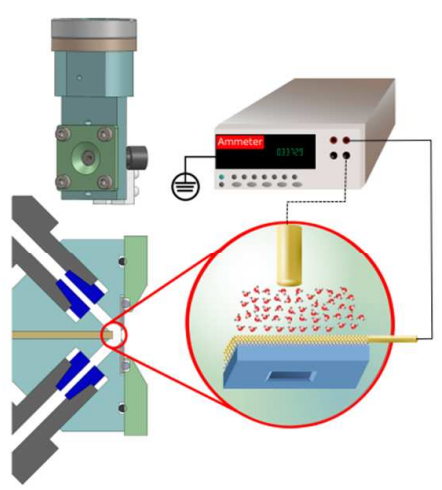


Studying the electronic structure of liquid water and aqueous solutions by soft X-rays has attracted much attention in recent years, ${ }^{1-6}$ and continues to be a vital research field. Resonant excitation by $\mathrm{X}$-rays is highly element-specific, which makes X-ray absorption spectroscopy (XAS) a widely used tool in many scientific disciplines. Due to the vacuum requirement for soft X-ray propagation, detection of XA spectra for liquid (volatile) samples in vacuum is very challenging. One of the most applied techniques to introduce liquid samples into a vacuum chamber is a liquid flow-cell with an ultra-thin membrane separating the liquid from the vacuum. ${ }^{7,8}$ When equipped with multiple electrodes, such a liquid cell can act as a standard electrochemical cell. It is therefore of great interest to combine XAS and the liquid cell technique for in situ/operando investigations on liquid-based materials. ${ }^{9-12}$

When a sample's thickness exceeds the penetration depth of soft X-rays, which is the case for the liquid cell adopted in this study, detection of XA spectra in the transmission mode is not applicable. Therefore, fluorescence yield (FY) or electron yield (EY) must be employed to acquire XA spectra. Due to the significant thickness of typical membranes, e.g. $\mathrm{Si}_{3} \mathrm{~N}_{4}$ and $\mathrm{SiC}$ membranes $(\sim 100 \mathrm{~nm})$, the electrons created within liquid solutions cannot penetrate the membrane and escape into vacuum. The FY was thus considered the only feasible way to probe the liquid-phase species behind membranes. However, EY has been recently realized in liquid cell studies, thanks to the newly developed graphene membranes. ${ }^{13}$ Another recently developed detection method in liquid cells is to measure the electric current induced by X-ray excitation. This electric-current detection which was categorized as total electron yield (TEY) ${ }^{9-11}$ is further explored and discussed in detail in this study. In ref. ${ }^{9}$, the synchrotron X-ray source was frequency-modulated before applied to the liquid cell, and the resulting electric current that matched the modulation frequency was then collected by an Aucoated $\mathrm{Si}_{3} \mathrm{~N}_{4}$ membrane. By this lock-in technique the large background signal originating from the continuous ionic current was excluded from the measurements.

The ionic current, which was discarded in previous studies as an unwanted background, is however found to be useful, and thus exploited in this study. We demonstrate that without modulation of X-ray pulse frequency the O K-edge and Fe L-edge XA spectra of liquid samples behind the membrane can still be obtained by measuring the ionic current through the liquid cell with two electrodes connecting to an ammeter, as illustrated in figure 1. Because the solvated ions are the charge carriers that produce the current signal (discussed below), this two-electrode detection method is termed here as total ion yield (TIY). If only the front electrode is connected to the ammeter, the charge flow is carried solely by electrons (discussed below). This one-electrode detection corresponds to the traditional drain-current 
measurement that is often applied to conductive solid samples, ${ }^{14-16}$ and is thus referred to as total electron yield (TEY) in this study. It is noteworthy that the term TIY and ion-detection technique has already been proposed in previous XAS studies of liquid microjets. ${ }^{17-19}$ Those TIY measurements are however based on the one-electrode detection which is only applicable to windowless liquid samples due to the technique's surface sensitivity. The twoelectrode TIY detection introduced in this study is, on the other hand, applied in liquid cells with membrane windows. The distinction between the one- and two-electrode TIY detections becomes clear in the later discussions.

Au-coated $\mathrm{Si}_{3} \mathrm{~N}_{4}$ membranes with $\mathrm{Cr}$ buffer layer in between, purchased from Silson Ltd., were employed in the investigated liquid cell. The thicknesses of the individual layers of $\mathrm{Si}_{3} \mathrm{~N}_{4}, \mathrm{Cr}$ and $\mathrm{Au}$ in the membranes are 100,5 and $10 \mathrm{~nm}$, respectively. The chemicals iron(III) nitrate $\left(\mathrm{Fe}\left(\mathrm{NO}_{3}\right)_{3}\right)$ and acetonitrile $\left(\mathrm{CH}_{3} \mathrm{CN}\right)$ used in this study were purchased from Sigma-Aldrich with purity $\geq 99.9 \%$. Deionized water was used as sample and as solvent for $\mathrm{Fe}\left(\mathrm{NO}_{3}\right)_{3}$ aqueous solutions of 1 and $2 \mathrm{M}$ concentrations. The liquid cell is equipped with two electrodes for the current measurements. The Au-coated membrane serves as the front electrode that is exposed to the incident X-rays; and an Au rod of $1.6 \mathrm{~mm}$ diameter located at approximately $1 \mathrm{~mm}$ away from the front electrode acts as the back electrode. The current measurements were carried out by connecting an ammeter (Keithley 6514) either to the front electrode only (TEY detection) or to both the front and back electrodes (TIY detection), as depicted in figure 1. Unsteady current fluctuation was observed when the liquid was flowing inside the cell. Therefore, the liquid flow was stopped during the current measurements, but resumed between the measurements to refresh samples. Radiation damage to the non-flowing liquid samples is not observed; and the acquired XA spectra are consistent with the previous reports (see figures 4 and 5 and the related discussions). The XA spectra at the $\mathrm{O}$ K-edge, $\mathrm{Cr}$ L-edge and Fe L-edge were obtained by measuring TEY and TIY, as well as by detecting partial fluorescence yield (PFY), as illustrated in figure 1. The emitted X-ray photons from the $\mathrm{O} 2 \mathrm{p} \rightarrow 1 \mathrm{~s}, \mathrm{Cr} 3 \mathrm{~d} \rightarrow 2 \mathrm{p}$ and Fe $3 \mathrm{~d} \rightarrow 2 \mathrm{p}$ decay channels in the PFY detections are respectively collected along the polarization direction of the incident X-ray beam. The X-ray spectrometer employs a Rowland-circle-geometry with a grating of $13 \mathrm{~m}$ radius and 2400 lines per $\mathrm{mm}$. The photon detector consists of a micro-channel plate (MCP)/phosphorus screen/CCD camera stack. The experiment was conducted at the U49/2-PGM1 undulator beamline of BESSY II, Berlin, using the LiXEdrom experimental station which is described in detail elsewhere. ${ }^{20}$ 
Soft X-rays can penetrate typical liquid-cell membranes and reach up to $\sim 1 \mu \mathrm{m}$ into liquid samples, while the photoelectrons excited by X-ray absorption travel only a few nanometers in samples due to their short inelastic-mean-free-path (IMFP). ${ }^{21}$ The photoelectrons that are created in the bulk solution cannot reach either of the electrodes and therefore do not contribute to the current measurements. However, the photoelectrons created in the vicinity of the front electrode (10-nm-thick Au film) can be collected by this electrode, which may result in an electric current detectable by the ammeter. ${ }^{9}$ To test this idea the $\mathrm{O} \mathrm{K}$ edge XA spectra have been measured from the liquid cell filled with deionized water. In this setup, only the front electrode was connected to the grounded ammeter, leaving the back electrode electrically floating. The resulting TEY-XA spectrum is presented in figure 2, along with the corresponding PFY-XA spectrum for comparison. The bulk-sensitive PFY detection produces the characteristic water absorption features - II at $535 \mathrm{eV}$, and III at $537 \mathrm{eV}$ which are known to be related to the unsaturated hydrogen bonds in the bulk water. ${ }^{6,9,22}$ The feature I of the PFY-XA spectrum, located at $531 \mathrm{eV}$, is absent in the XA spectra of neat liquid water. ${ }^{6,9,22}$ This feature is believed to be from either the adsorbed oxygen species containing $\mathrm{C}=\mathrm{O}$ double bond ${ }^{23,24}$ on the membrane surface or the embedded oxygen compounds, probably $\mathrm{CrO}_{\mathrm{x}}{ }^{25}$ formed during the $\mathrm{Cr}$ deposition process, within the membrane. The TEYXA spectrum at the O K-edge exhibits much suppressed bulk-solution features II and III, while much enhanced surface (or embedded) feature I with respect to the PFY spectrum. This intensity variation is reasonable since TEY is not sensitive to the bulk water species, but only to the oxygen-containing species at the membrane surface, and perhaps to the species close to the $\mathrm{Au}$ film as well. When replacing water by acetonitrile $\left(\mathrm{CH}_{3} \mathrm{CN}\right)$ which contains no oxygen, both TEY- and PFY-XA spectra in the lower part of figure 2 exhibit no features II and III, but a strong feature I, similar to the TEY-XA spectrum in the upper part, which is expected because the contribution of the bulk-water species is absent in all three spectra. There is an intensity difference of the feature I between the TEY- and PFY-XA spectra of $\mathrm{CH}_{3} \mathrm{CN}$, indicating that the respective signals may originate from different oxygen sources. With the bulk water species excluded the adsorbed oxygen species at the membrane surface and the embedded oxygen species within the membrane could still contribute to the PFY and TEY measurements. PFY detection shall be able to pick up the signals from both oxygen sources, while TEY may be sensitive to only one of them, which becomes clear in the following discussion for figure 3. Besides the possible sources of oxygen signal, the different decay channels employed by PFY and TEY detections may affect the intensity as well, as discussed previously in ref. ${ }^{26,27}$. 
It is difficult to identify the exact oxygen source that contributes to the TEY signals because oxygen contamination is generally present on the membrane surface and within the membrane. The Cr layer which exists only inside the membrane, however, provides a perfect candidate to test whether the TEY detection is capable of picking up signals from the embedded species within the membrane. Cr was therefore selected as the element to be investigated by measuring the TEY- and PFY-XA spectra at the Cr L-edge, as shown in figure 3. The Cr L-edge XA spectra in the upper panel of figure 3 were obtained from the membrane installed in the liquid cell filled with deionized water. The PFY-XA spectrum exhibits, as expected, two pronounced peaks representing the $\mathrm{Cr}_{3}$ and $\mathrm{L}_{2}$ edges. $^{28,29}$ The TEY-XA spectrum that was recorded simultaneously as the PFY-XA spectrum, on the other hand, presents a flat line with no distinguishable $\mathrm{Cr}$ features, suggesting that the embedded species is not detectable by the TEY method. The TEY investigation of a standalone membrane by measuring sample drain current, as shown in the lower panel of figure 3 , further corroborates this conclusion.

When a standalone membrane is mounted on a solid sample holder (which is a $\mathrm{Cu}$ plate with a through-hole) with the 10-nm-thick Au film facing the incident X-rays, the TEY detection gives rise to the expected Cr L-edge XA spectrum shown as the top trace in the lower panel of figure 3. The incident X-rays can easily penetrate the 10-nm-thick Au film and reach the $\mathrm{Cr}$ layer, and some of the excited photoelectrons from the $\mathrm{Cr}$ layer are able to escape through the Au film into the vacuum. The remaining photoholes are then compensated by the electrons moving in the direction of ground $\rightarrow$ ammeter $\rightarrow$ membrane, producing the TEY signal. When the membrane on the $\mathrm{Cu}$ plate was flipped, i.e. the $\mathrm{Si}_{3} \mathrm{~N}_{4}$ window facing the vacuum and $\mathrm{Au}$ film facing the through-hole of the $\mathrm{Cu}$ plate, the TEY signal strength is reduced, as shown in figure 3 . In this situation, the X-ray flux is attenuated by the $100-\mathrm{nm}$ thick $\mathrm{Si}_{3} \mathrm{~N}_{4}$ film whose thickness exceeds the IMFP of photoelectrons significantly. Nevertheless, the escaping photoelectrons from the Cr layer are still able to go through the 10 -nm-thick $\mathrm{Au}$ film into the vacuum by passing the through-hole in the $\mathrm{Cu}$ plate. However, when a $0.1-\mathrm{mm}$-thick $\mathrm{Au}$ plate is inserted between the $\mathrm{Au}$ film of the membrane and the bottom $\mathrm{Cu}$ plate, the escaping path for the excited photoelectrons is completely blocked, and no photoelectron that is created within the Cr layer can leave the membrane. Therefore, no $\mathrm{Cr}$ L-edge XA spectrum was detected, as observed in the lower panel of figure 3, even though the incident $\mathrm{X}$-rays can reach the $\mathrm{Cr}$ layer and generate photoelectrons in the vicinity of the $\mathrm{Au}$ film in this situation. Figures 2 and 3 demonstrate that the TEY detection (with the front 
electrode connected to the ammeter) that utilizes electron as the charge carrier is only sensitive to the species at very surface where the excited photoelectrons can escape into the vacuum. ${ }^{14-16}$ The acquired TEY-XA spectra at the O K-edge (figure 2) and Cr L-edge (traces in figure 3 with visible $\mathrm{Cr}$ XA features) are from the surface-adsorbed oxygen species, and the embedded $\mathrm{Cr}$ layer that is only nanometers away from the surface, respectively.

It is now clear that the TEY detection, with one single electrode connected to the ammeter, only probes the surface species at the vacuum side. It is however surprising to observe that adding the back electrode of the liquid cell to the current measurements dramatically changes the measurement outcome. When both the front and back electrodes in the water-filled liquid cell are connected to the ammeter, the measured current strength at the O K-edge is boosted 40 times higher with respect to the one-electrode TEY measurement, and the resulting XA spectrum becomes very similar to the PFY-XA spectrum, as well as distinctive from the TEY-XA spectrum, as shown in figure 4. The middle spectrum in figure 4 has clear features II and III, and almost no feature I, indicating that the two-electrode detection only probes the liquid water behind the membrane, eliminating any contributions from both adsorbed and embedded oxygen species. Since neither the incident X-rays nor the excited photoelectrons can reach the back electrode, it is quite intriguing that the inclusion of the back electrode can have such a profound influence on the current measurement. The incident X-rays exciting a sample actually always create electron - ion pairs upon photoionization, but only the electrons have been generally exploited so far as the charge carrier formulating the TEY detection. The utilization of the corresponding ions is much less explored, ${ }^{17-19}$ especially for solid sample measurements due to the immobility of the ions. The ions become, however, quite mobile in liquid phase, and the ionic-current that was previously considered as background noise may actually be useful to generate XA signals. We hence hypothesize that it is the ions induced by X-ray absorption that act as the charge carrier traveling towards the back electrode and constituting the detected current. The charge carrier in the deionized water could be the ions created during the EY process upon X-ray absorption and/or the neighboring water molecules next to the absorption site ionized by the energetic photoelectrons. The two-electrode detection scheme is therefore termed as total ion yield (TIY), as indicated in figure 4. The mobility and long-distance transportability of the ions in liquid may provide an indispensable assistance to realize the charge flow through the bulk liquid. This long-distance transport cannot be achieved by electrons because of their extremely short IMFP. The previously reported TIY measurements on liquid microjets ${ }^{17-19}$ are essentially one-electrode detections which require the electrons or ions produced upon 
photoionization to escape from the investigated samples into vacuum (sometimes with the assistance of electric bias). Therefore, their probing depth is limited to the length of the electron or ion escape path, similar to the above-discussed TEY detections. The one-electrode TIY detection, although utilizing ions as charge carriers as well, is different from the twoelectrode TIY applied here, and is not suitable to probe liquid solutions behind membranes in liquid cells.

Since the ions are created within the $\sim 1 \mu \mathrm{m}$ region from the front electrode and the back electrode is located about $1 \mathrm{~mm}$ away, diffusion time is expected to play a role in the ion transport between the two electrodes. Consequently, the TIY detection may show a delayed response to the X-ray absorption when compared to the PFY detection, which could lead to a universal energy shift of all TIY-XA peaks towards higher energy with respect to the corresponding peak positions in the PFY-XA spectra. Such a postulated peak shift is, however, not observed in figure 4, as well as not in the following figure 5 . Considering that the data acquisition time at each excitation energy was set to $2 \mathrm{~s}$ for all the XA measurements, we can estimate that the diffusion time required for the ion transport through the liquid cell must be less than $2 \mathrm{~s}$.

The ions in the deionized water are created upon X-ray absorption. No pre-existing ions are supposed to be present. It would be interesting to see if the TIY measurements can also be applied to salt solutions with pre-existing ions. The TIY detection was then tested for 1 and $2 \mathrm{M}$ concentrations of $\mathrm{Fe}\left(\mathrm{NO}_{3}\right)_{3}$ aqueous solutions, where positive and negative ions pre-exist in the liquid, to measure the Fe L-edge XA spectra. The obtained TIY-XA spectra are compared with the corresponding PFY-XA spectra that were recorded simultaneously, as shown in figure 5. Compared to the PFY-XA spectra, the TIY-XA spectra exhibit much enhanced first feature of $\mathrm{Fe}(\mathrm{III})$ at about $709 \mathrm{eV}$ and much reduced intensity at the $\mathrm{L}_{2}$ edge. There are various distortion effects in the XAS measurements that collect relaxation emissions (FY or EY) from various decay channels. ${ }^{30-34}$ The well-known saturation effect normally enhances the $\mathrm{L}_{2}$ edge intensity relative to the $\mathrm{L}_{3}$ edge for transition metal L-edge XA spectra. ${ }^{30,31,33,34}$ For the Fe(III) L-edge XA spectra, the relative intensity of the first feature at $709 \mathrm{eV}$ with reference to the highest peak at $710 \mathrm{eV}$ is often reduced when the $\mathrm{Fe}$ $3 \mathrm{~d}$ valence orbitals are involved in the decay channels of FY or EY detections, as discussed previously in ref. ${ }^{27}$. One decay channel Fe $3 s \rightarrow 2 p$ was however determined to be free from all distortion effects and able to produce an undistorted Fe(III) L-edge XA spectrum. ${ }^{27}$ The undistorted $\mathrm{Fe}$ L-edge XA spectrum of $1 \mathrm{M} \mathrm{FeCl}_{3}$ aqueous solution acquired from this decay 
channel is thus superimposed onto the TIY-XA spectrum of $1 \mathrm{M} \mathrm{Fe}\left(\mathrm{NO}_{3}\right)_{3}$ aqueous solution for comparison, as shown in figure 5a. The comparison indicates that the TIY detection gives rise to the similarly reduced $\mathrm{L}_{2}$ edge and identical intensity ratio between the first $(709 \mathrm{eV})$ and second $(710 \mathrm{eV})$ absorption peaks with respect to the PFY $3 \mathrm{~s} \rightarrow 2 \mathrm{p}$ XA spectrum. The perfect match, especially for the intensity ratio of the two leading absorption features, between the benchmark Fe(III) L-edge XA spectrum taken previously from the PFY $3 \mathrm{~s} \rightarrow 2 \mathrm{p}$ decay channel and the TIY-XA spectrum acquired in this study strongly suggests that the TIY is a promising detection variant to obtain undistorted XA spectra for liquid samples.

The higher concentration of $2 \mathrm{M} \mathrm{Fe}\left(\mathrm{NO}_{3}\right)_{3}$ aqueous solution does not lead to significant differences in the XA spectra when compared to the spectra of the $1 \mathrm{M}$ solution, but only slightly enhances the first peak at $709 \mathrm{eV}$ and $\mathrm{L}_{2}$ edges for both PFY and TIY detections, as shown in the lower part of figure 5. These intensity increases could be related to the saturation effect which becomes more severe with higher concentration. ${ }^{31}$ The saturation effect always reduces the most intense peak, ${ }^{30-32}$ such as the peak at about $710 \mathrm{eV}$ of the Fe L-edge XA spectra in figure 5, which consequently leads to a relative enhancement of other peaks.

The detected TIY currents of $\mathrm{Fe}\left(\mathrm{NO}_{3}\right)_{3}$ aqueous solutions have the background levels at $\sim 100 \mathrm{nA}$ and the magnitudes of the most protruding peaks at $710 \mathrm{eV}$, relative to the background signal, within the range of $\sim 10 \mathrm{nA}$, which are quite robust and reproducible. The same TIY detections were attempted on the $1 \mathrm{M} \mathrm{FeCl}_{3}$ aqueous solution as well. The background current of the $\mathrm{FeCl}_{3}$ solution is, however, raised close to $10 \mu \mathrm{A}$, and no $\mathrm{Fe}(\mathrm{III}) \mathrm{L}-$ edge XA feature is observed. The negative $\mathrm{Cl}^{-}$ions which do not participate in the resonant $\mathrm{X}$-ray absorption process are suspected to be responsible for the absence of the Fe XA features. We speculate that the higher ionic background current is caused by the $\mathrm{Cl}^{-}$ions being smaller, lighter and hence more mobile than the $\mathrm{NO}_{3}{ }^{-}$ions in solution, which consequently inhibits the weak current variation induced by the X-rays from being detected.

In conclusion, the electric-current measurements have been conducted on a liquid cell equipped with two electrodes, and the resulting XA spectra at the O K-edge, Cr L-edge and Fe L-edge are compared with the spectra acquired by the bulk-sensitive PFY measurements. The one-electrode TEY detection has been determined to be exclusively surface sensitive, while the two-electrode TIY detection is found capable of eliminating the contributions from the adsorbed and embedded species on/in the membrane, and just probing the liquid species behind the membrane. The mobile ions in the liquid solutions are hypothesized to be the 
charge carriers constituting the detected current signals, and the resulting TIY-XA spectra can be obtained with or without pre-existing ions in solution. For Fe(III) complexes in solution, the TIY detection produced an undistorted XA spectrum at the Fe L-edge, suggesting its promising role in the XAS measurements for liquid samples. The TIY signals detected in this study are identified from the liquid-phase species behind the membrane. However, the exact location of the signal generation - at the solution/membrane interface, in the bulk solution, or the combination of both - cannot be unequivocally pinpointed, which remains to be investigated in the future.

\section{Acknowledgments}

This work is supported by the Deutsche Forschungsgemeinschaft (DFG) with grant No. 0420541101.

\section{References}

(1) Fransson, T.; Harada, Y.; Kosugi, N.; Besley, N. A.; Winter, B.; Rehr, J. J.; Pettersson, L. G. M.; Nilsson, A. X-Ray and Electron Spectroscopy of Water. Chem. Rev. 2016, 116, 7551-7569.

(2) Wernet, P.; Nordlund, D.; Bergmann, U.; Cavalleri, M.; Odelius, M.; Ogasawara, H.; Näslund, L. Å.; Hirsch, T. K.; Ojamäe, L.; Glatzel, P.; et al. The Structure of the First Coordination Shell in Liquid Water. Science 2004, 304, 995-999.

(3) Engel, N.; Atak, K.; Lange, K. M.; Gotz, M.; Soldatov, M.; Golnak, R.; Suljoti, E.; Rubensson, J.-E.; Aziz, E. F. DMSO-Water Clustering in Solution Observed in Soft XRay Spectra. J. Phys. Chem. Lett. 2012, 3, 3697-3701.

(4) Golnak, R.; Atak, K.; Suljoti, E.; Hodeck, K. F.; Lange, K. M.; Soldatov, M. A.; Engel, N.; Aziz, E. F. Local Electronic Structure of Aqueous Zinc Acetate: Oxygen K-Edge XRay Absorption and Emission Spectroscopy on Micro-Jets. Phys. Chem. Chem. Phys. 2013, 15, 8046-8049.

(5) Winter, B. Interfaces: Scientists Strike Wet Gold. Nat. Chem. 2015, 7 (3), 192-194.

(6) Smith, J. D.; Cappa, C. D.; Messer, B. M.; Drisdell, W. S.; Cohen, R. C.; Saykally, R. J. Probing the Local Structure of Liquid Water by X-Ray Absorption Spectroscopy. $J$. Phys. Chem. B 2006, 110, 20038-20045.

(7) Fuchs, O.; Maier, F.; Weinhardt, L.; Weigand, M.; Blum, M.; Zharnikov, M.; Denlinger, J.; Grunze, M.; Heske, C.; Umbach, E. A Liquid Flow Cell to Study the Electronic Structure of Liquids with Soft X-Rays. Nucl. Instrum. Methods Phys. Res. Sect. Accel. Spectrometers Detect. Assoc. Equip. 2008, 585, 172-177.

(8) Guo, J.-H.; Luo, Y.; Augustsson, A.; Rubensson, J.-E.; Såthe, C.; Ågren, H.; Siegbahn, H.; Nordgren, J. X-Ray Emission Spectroscopy of Hydrogen Bonding and Electronic Structure of Liquid Water. Phys. Rev. Lett. 2002, 89, 137402.

(9) Velasco-Velez, J.-J.; Wu, C. H.; Pascal, T. A.; Wan, L. F.; Guo, J.; Prendergast, D.; Salmeron, M. The Structure of Interfacial Water on Gold Electrodes Studied by X-Ray Absorption Spectroscopy. Science 2014, 346, 831-834. 

Phenom. 2015, 200, 264-273.

(11) Wu, C. H.; Weatherup, R. S.; Salmeron, M. B. Probing Electrode/Electrolyte Interfaces in Situ by X-Ray Spectroscopies: Old Methods, New Tricks. Phys. Chem. Chem. Phys. 2015, 17, 30229-30239.

(12) Bora, D. K.; Glans, P.-A.; Pepper, J.; Liu, Y.-S.; Du, C.; Wang, D.; Guo, J.-H. An UltraHigh Vacuum Electrochemical Flow Cell for in Situ/Operando Soft X-Ray Spectroscopy Study. Rev. Sci. Instrum. 2014, 85, 043106.

(13) Velasco-Velez, J. J.; Pfeifer, V.; Hävecker, M.; Weatherup, R. S.; Arrigo, R.; Chuang, C.-H.; Stotz, E.; Weinberg, G.; Salmeron, M.; Schlögl, R.; et al. Photoelectron Spectroscopy at the Graphene-Liquid Interface Reveals the Electronic Structure of an Electrodeposited Cobalt/Graphene Electrocatalyst. Angew. Chem. Int. Ed. 2015, 54, 14554-14558.

(14) Frazer, B. H.; Gilbert, B.; Sonderegger, B. R.; De Stasio, G. The Probing Depth of Total Electron Yield in the Sub-keV Range: TEY-XAS and X-PEEM. Surf. Sci. 2003, 537, 161-167.

(15) Kasrai, M.; Lennard, W. N.; Brunner, R. W.; Bancroft, G. M.; Bardwell, J. A.; Tan, K. H. Sampling Depth of Total Electron and Fluorescence Measurements in Si L- and KEdge Absorption Spectroscopy. Appl. Surf. Sci. 1996, 99, 303-312.

(16) Schroeder, S. L. M.; Moggridge, G. D.; Ormerod, R. M.; Rayment, T.; Lambert, R. M. What Determines the Probing Depth of Electron Yield XAS? Surf. Sci. 1995, 324, L371-L377.

(17) Wilson, K. R.; Tobin, J. G.; Ankudinov, A. L.; Rehr, J. J.; Saykally, R. J. Extended XRay Absorption Fine Structure from Hydrogen Atoms in Water. Phys. Rev. Lett. 2000, 85, 4289-4292.

(18) Wilson, K. R.; Rude, B. S.; Catalano, T.; Schaller, R. D.; Tobin, J. G.; Co, D. T.; Saykally, R. J. X-Ray Spectroscopy of Liquid Water Microjets. J. Phys. Chem. B 2001, 105, 3346-3349.

(19) Lam, R. K.; Shih, O.; Smith, J. W.; Sheardy, A. T.; Rizzuto, A. M.; Prendergast, D.; Saykally, R. J. Electrokinetic Detection for X-Ray Spectra of Weakly Interacting Liquids: N-Decane and N-Nonane. J. Chem. Phys. 2014, 140, 234202.

(20) Aziz, E. F.; Xiao, J.; Golnak, R.; Tesch, M. LiXEdrom: High Energy Resolution RIXS Station Dedicated to Liquid Investigation at BESSY II. J. Large-Scale Res. Facil. JLSRF 2016, $2,80$.

(21) Thürmer, S.; Seidel, R.; Faubel, M.; Eberhardt, W.; Hemminger, J. C.; Bradforth, S. E.; Winter, B. Photoelectron Angular Distributions from Liquid Water: Effects of Electron Scattering. Phys. Rev. Lett. 2013, 111, 173005.

(22) Lange, K. M.; Könnecke, R.; Soldatov, M.; Golnak, R.; Rubensson, J.-E.; Soldatov, A.; Aziz, E. F. On the Origin of the Hydrogen-Bond-Network Nature of Water: X-Ray Absorption and Emission Spectra of Water-Acetonitrile Mixtures. Angew. Chem. 2011, 123, 10809-10813.

(23) Ottosson, N.; Aziz, E. F.; Bradeanu, I. L.; Legendre, S.; Öhrwall, G.; Svensson, S.; Björneholm, O.; Eberhardt, W. An Electronic Signature of Hydrolysation in the X-Ray Absorption Spectrum of Aqueous Formaldehyde. Chem. Phys. Lett. 2008, 460, 540-542.

(24) Schreck, S.; Pietzsch, A.; Kennedy, B.; Såthe, C.; Miedema, P. S.; Techert, S.; Strocov, V. N.; Schmitt, T.; Hennies, F.; Rubensson, J.-E.; et al. Ground State Potential Energy Surfaces around Selected Atoms from Resonant Inelastic X-Ray Scattering. Sci. Rep. 2016, 7, 20054. 
(25) de Groot, F. M. F.; Grioni, M.; Fuggle, J. C.; Ghijsen, J.; Sawatzky, G. A.; Petersen, H. Oxygen 1s X-Ray-Absorption Edges of Transition-Metal Oxides. Phys. Rev. B 1989, 40, 5715-5723.

(26) Golnak, R.; Bokarev, S. I.; Seidel, R.; Xiao, J.; Grell, G.; Atak, K.; Unger, I.; Thürmer, S.; Aziz, S. G.; Kühn, O.; et al. Joint Analysis of Radiative and Non-Radiative Electronic Relaxation Upon X-Ray Irradiation of Transition Metal Aqueous Solutions. Sci. Rep. 2016, 6, 24659.

(27) Golnak, R.; Xiao, J.; Atak, K.; Unger, I.; Seidel, R.; Winter, B.; Aziz, E. F. Undistorted $\mathrm{X}$-Ray Absorption Spectroscopy Using S-Core-Orbital Emissions. J. Phys. Chem. A 2016, 120, 2808-2814.

(28) Meyers, D.; Mukherjee, S.; Cheng, J.-G.; Middey, S.; Zhou, J.-S.; Goodenough, J. B.; Gray, B. A.; Freeland, J. W.; Saha-Dasgupta, T.; Chakhalian, J. Zhang-Rice Physics and Anomalous Copper States in A-Site Ordered Perovskites. Sci. Rep. 2013, 3, 1834.

(29) Zhang, K. H. L.; Du, Y.; Sushko, P. V.; Bowden, M. E.; Shutthanandan, V.; Sallis, S.; Piper, L. F. J.; Chambers, S. A. Hole-Induced Insulator-to-Metal Transition in La1-xSrxCrO3 Epitaxial Films. Phys. Rev. B 2015, 91, 155129.

(30) Achkar, A. J.; Regier, T. Z.; Wadati, H.; Kim, Y.-J.; Zhang, H.; Hawthorn, D. G. Bulk Sensitive X-Ray Absorption Spectroscopy Free of Self-Absorption Effects. Phys. Rev. B 2011, 83, 081106.

(31) Eisebitt, S.; Böske, T.; Rubensson, J.-E.; Eberhardt, W. Determination of Absorption Coefficients for Concentrated Samples by Fluorescence Detection. Phys. Rev. B 1993, 47, 14103-14109.

(32) Achkar, A. J.; Regier, T. Z.; Monkman, E. J.; Shen, K. M.; Hawthorn, D. G. Determination of Total X-Ray Absorption Coefficient Using Non-Resonant X-Ray Emission. Sci. Rep. 2011, 1, 182.

(33) Tröger, L.; Arvanitis, D.; Baberschke, K.; Michaelis, H.; Grimm, U.; Zschech, E. Full Correction of the Self-Absorption in Soft-Fluorescence Extended X-Ray-Absorption Fine Structure. Phys. Rev. B 1992, 46, 3283-3289.

(34) Błachucki, W.; Szlachetko, J.; Hoszowska, J.; Dousse, J.-C.; Kayser, Y.; Nachtegaal, M.; Sá, J. High Energy Resolution Off-Resonant Spectroscopy for X-Ray Absorption Spectra Free of Self-Absorption Effects. Phys. Rev. Lett. 2014, 112, 173003. 


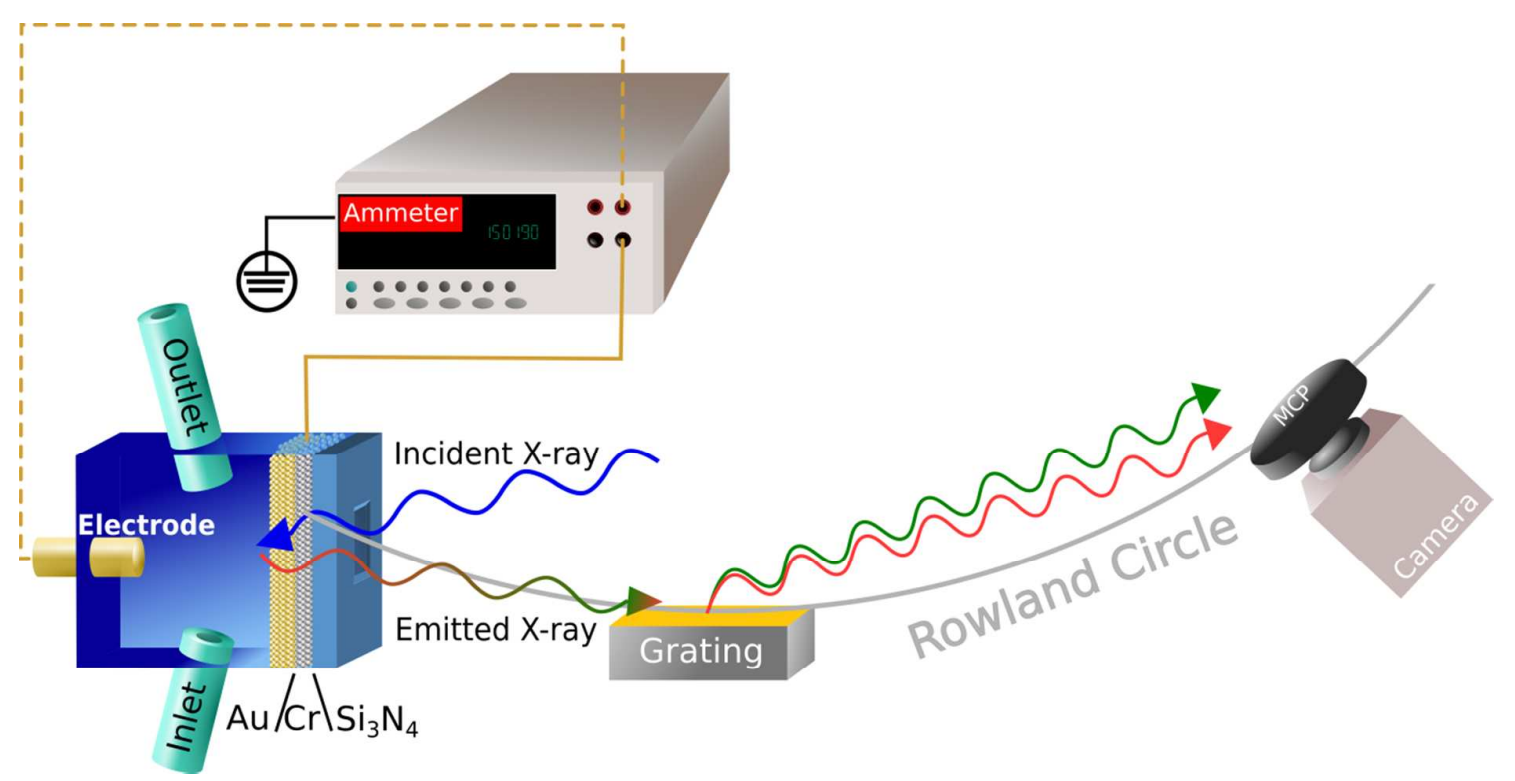

Figure 1. The detection schemes of electric-current and fluorescence measurements under Xray illumination: (1) a Keithley ammeter with one connection (solid wire) to the Au-coated membrane (front electrode) as the TEY detection scheme, (2) the same ammeter with two connections (solid and dashed wires) to the front and back electrodes as the TIY scheme, and (3) the emitted X-ray photons dispersed by a grating and detected by the $\mathrm{MCP} /$ phosphorus screen/camera stack as the PFY measurement. The thicknesses of the individual layers that constitute the membrane assembly $\mathrm{Au} / \mathrm{Cr} / \mathrm{Si}_{3} \mathrm{~N}_{4}$ are $10 / 5 / 100 \mathrm{~nm}$, respectively. The distance between the front and back electrodes is about $1 \mathrm{~mm}$. 


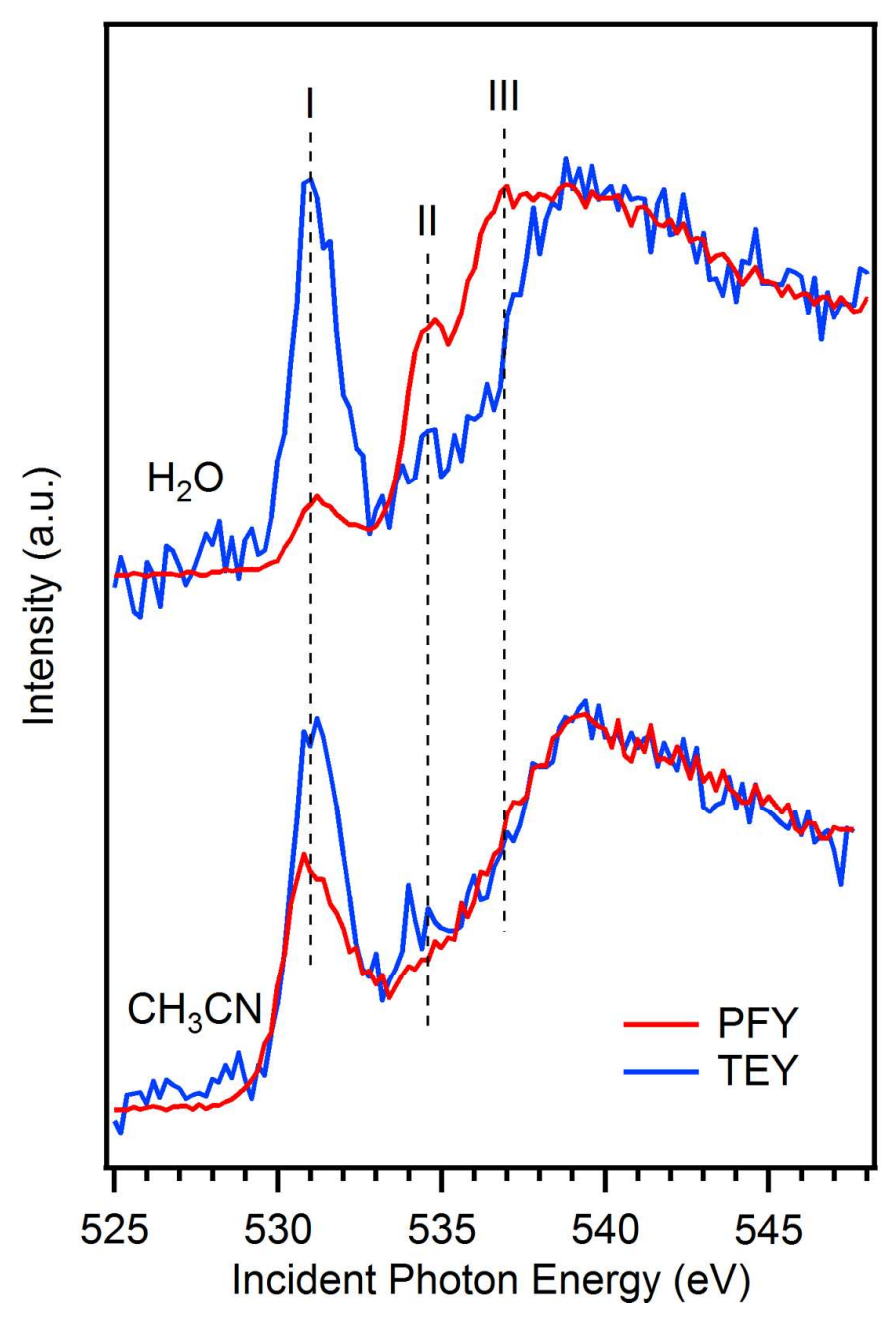

Figure 2. The O K-edge PFY- and TEY-XA spectra taken from the liquid cell filled with deionized water (upper traces) and acetonitrile (lower traces). Three vertical dashed lines indicate three major absorption features I, II and III in the spectra. The PFY and TEY spectra are pair-wise normalized to their background signals. 


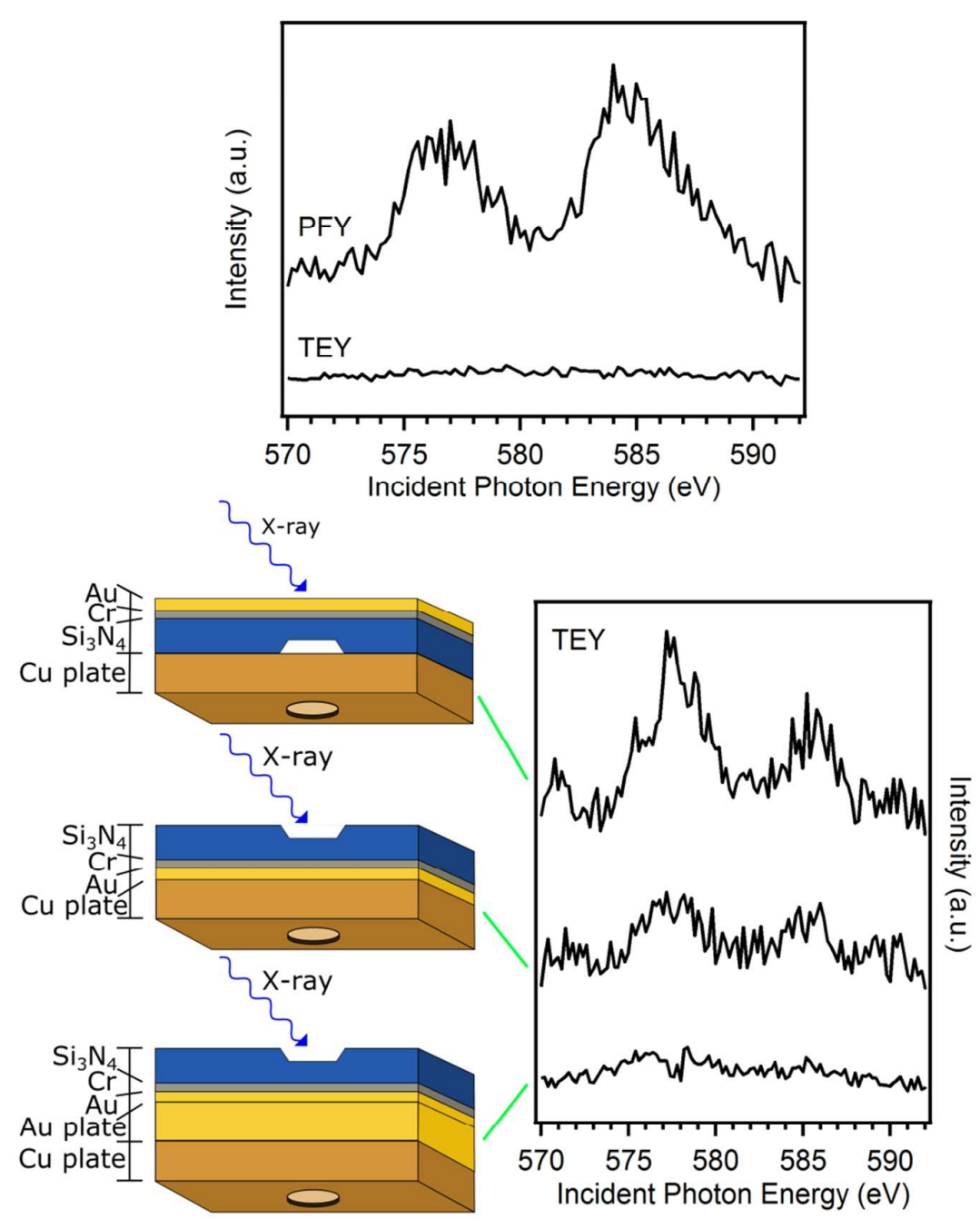

Figure 3. Upper panel: The Cr L-edge PFY- and TEY-XA spectra measured from a Aucoated membrane installed in the liquid cell filled with deionized water. Lower panel: the $\mathrm{Cr}$ L-edge TEY-XA spectra taken from a standalone membrane mounted on a $\mathrm{Cu}$ plate in three different configurations: (1) the 10-nm-thick Au film faces the incoming X-rays, while the 100-nm-thick $\mathrm{Si}_{3} \mathrm{~N}_{4}$ faces the $\mathrm{Cu}$ plate (top), (2) the $\mathrm{Si}_{3} \mathrm{~N}_{4}$ film faces the X-rays, and the $\mathrm{Au}$ film faces the $\mathrm{Cu}$ plate (middle), and (3) the $\mathrm{Si}_{3} \mathrm{~N}_{4}$ faces the X-rays with a $0.1-\mathrm{mm}$-thick $\mathrm{Au}$ plate inserted between the $10-\mathrm{nm}$-thick $\mathrm{Au}$ film and the $\mathrm{Cu}$ plate (bottom). There is a through-hole in the $\mathrm{Cu}$ plate that is always aligned with the membrane window. The membrane top surface that faces the vacuum was electrically connected to the bottom $\mathrm{Cu}$ plate by adhesive/conductive $\mathrm{Cu}$ tapes, and the $\mathrm{Cu}$ plate was grounded through the Keithley ammeter. 


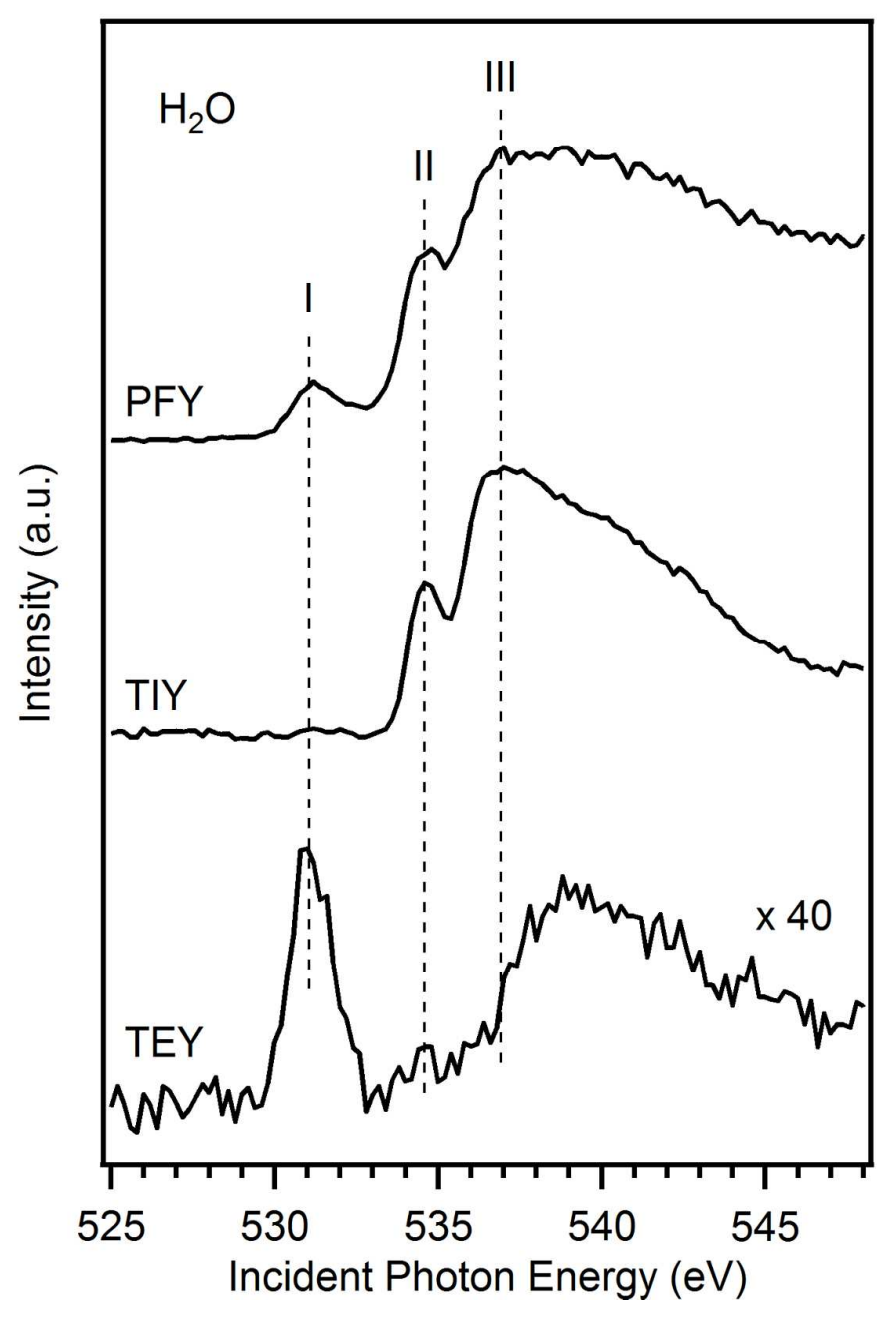

Figure 4. The O K-edge PFY-, TIY-, and TEY-XA spectra obtained from the liquid cell filled with deionized water. The TEY intensity is multiplied by 40 times in order to be comparable with the TIY intensity. The PFY- and TEY-XA spectra, as well as the markings I, II and III for the three major absorption features, are identical to those in figure 2. 


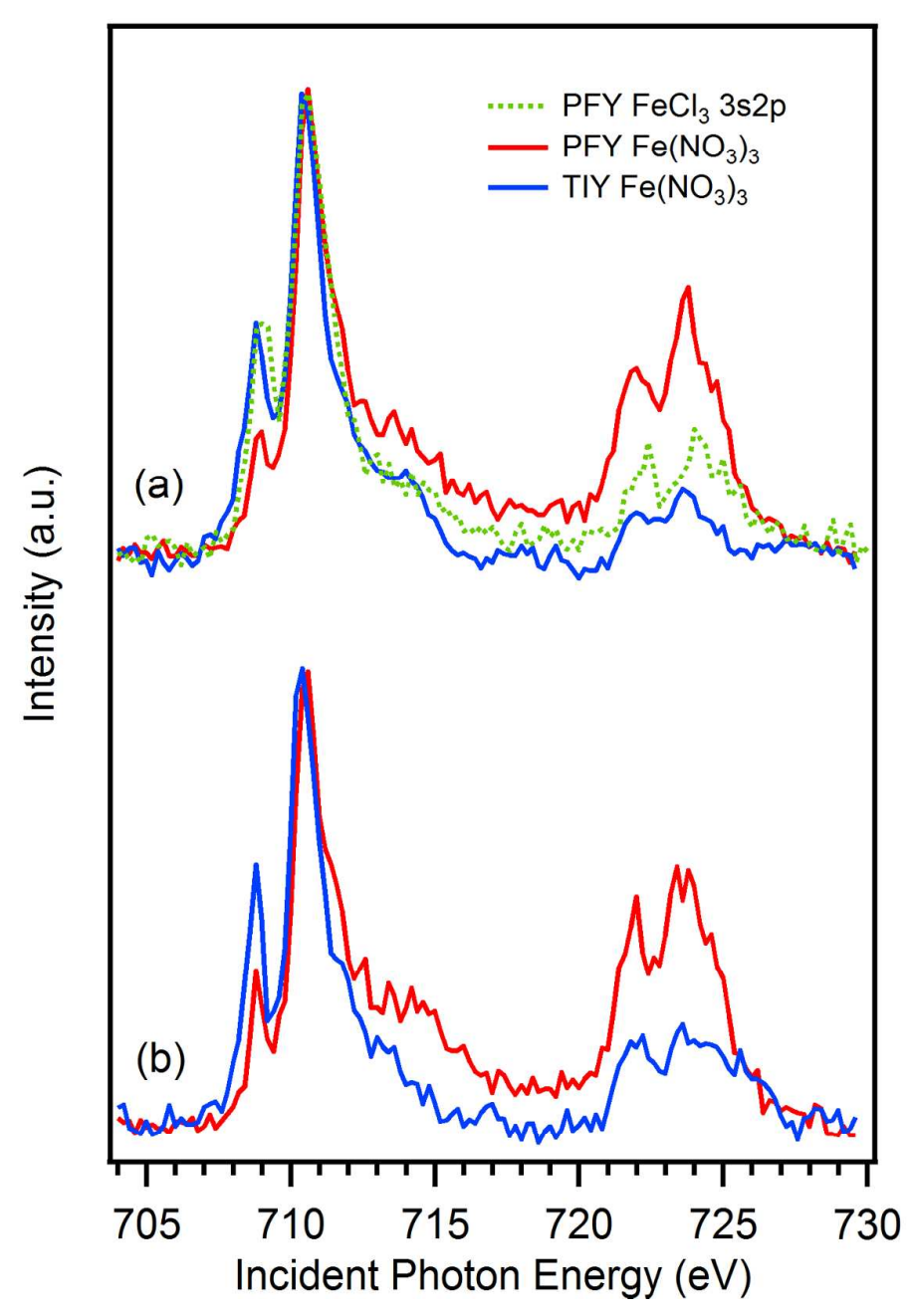

Figure 5. The Fe L-edge PFY- and TIY-XA spectra taken from the $1 \mathrm{M}$ (a) and $2 \mathrm{M}$ (b) concentrations of $\mathrm{Fe}\left(\mathrm{NO}_{3}\right)_{3}$ aqueous solutions in the liquid cell. The previously reported $\mathrm{Fe}(\mathrm{III}) \mathrm{L}$-edge PFY-XA spectrum of $1 \mathrm{M} \mathrm{FeCl}_{3}$ aqueous solution taken from the $\mathrm{Fe} 3 \mathrm{~s} \rightarrow 2 \mathrm{p}$ decay channel ${ }^{27}$ is superimposed in (a) for comparison. All spectra are normalized to the most intense absorption feature at around $710 \mathrm{eV}$. 